\title{
von Petz, Ursula (2016): Robert Schmidt 1869-1934. Stadtbaumeister in Essen und Landesplaner im Ruhrgebiet
}

\author{
Tübingen/Berlin: Ernst Wasmuth Verlag, 38 Abb., 240 S.
}

\section{Wendelin Strubelt ${ }^{1}$}

Online publiziert: 3. Mai 2017

(c) Springer-Verlag Berlin Heidelberg 2017

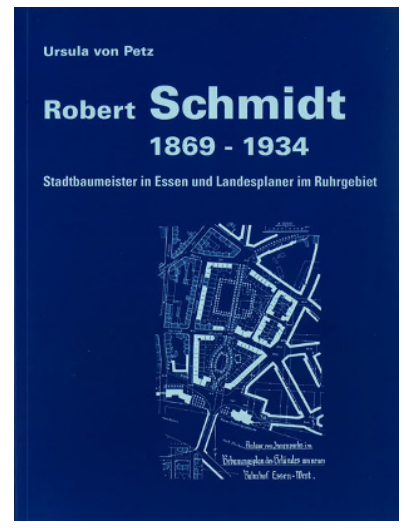

2012, zum 100. Jahrestag der Veröffentlichung der Denkschrift „Grundsätze zur Aufstellung eines General-Siedelungsplanes für den Regierungsbezirk Düsseldorf“" (Schmidt 1912), die Robert Schmidt zu einem der intellektuellen Gründungsväter der überörtlichen Planung - nicht nur in Deutschland - werden ließ, veranstaltete der Regionalverband Ruhr, die Nachfolgeorganisation des Siedlungsverbandes Ruhrkohlenbezirk (SVR), dessen erster Direktor Schmidt 1920 wurde, ein Symposion, das mit der Frage „Von Robert Schmidt lernen?“ die Nachhaltigkeit seines Wirkens thematisieren wollte. In der Dokumentation dieser Tagung (Kastorff-Viehmann/Utku/Regionalverband Ruhr 2014) ist auch ein kurzer Text von Ursula von Petz enthalten, der sich mit dem Werdegang und Wirken Robert Schmidts befasst. Jetzt hat sie mit dem zu rezensierenden Buch eine umfangreiche Würdigung seines Wirkens vorge-

Dr. Wendelin Strubelt strubelt@uni-bonn.de

1 Bundesamt für Bauwesen und Raumordnung i.R., Schloßstraße 29, 53115 Bonn, Deutschland legt. Es ist eine umfangreiche, materialreiche Würdigung entstanden, wie es sie bisher trotz Schmidts unumstrittener Leistungen und Bedeutung erstaunlicherweise noch nicht gegeben hat.

Ursula von Petz hat keine Biographie im herkömmlichen Sinne geschrieben, sondern eher eine umfangreiche Dokumentation seiner intellektuellen und planerischen Spuren, eine detailreiche Schilderung seines Lebens im Spiegel der Quellen, die über ihn und zu ihm überhaupt zur Verfügung stehen. Denn da seine persönlichen Unterlagen (Korrespondenzen, Notizen, Manuskripte, private Bibliothek), aber auch die Unterlagen des Siedlungsverbandes im Zweiten Weltkrieg in Flammen aufgingen (S. 22 f.), fehlen ausreichende Quellen, um eine umfassende Biographie zu verfassen, wie sie sie gerne im Vergleich zu einer vorliegenden Biographie über Werner Hegemann geschrieben hätte. ${ }^{1}$ Aber sie möchte ihr Buch auch nicht als ein Findebuch oder als „Reader“ verstanden wissen, sondern durchaus als den Versuch einer umfassenden Biographie (S. 8).

Sie beginnt sie mit einer Zusammenstellung von ,Urteilen' über Robert Schmidt aus dem Kreis seiner ,Kollegen und Wegbegleiter‘. Neben den bekannten, fast geläufigen positiven Einschätzungen, wie ,lebensprühend“, ,genialer Städtebauer und Verbandsdirektor" gibt es aber auch Aussagen wie: „Robert Schmidt war in seinem Fach sicherlich ein ganz großer Mann; aber auch große Männer bestehen nicht nur aus Gutem, sondern haben auch ihre Fehler und deren hatte Robert Schmidt besonders in charakterlicher Hinsicht nicht wenige“ (S. 15). Auch wenn diese Einschätzung von

\footnotetext{
${ }^{1}$ Vgl. das von Ursula von Petz explizit genannte Vorbild einer ,biographie intellectuelle" von Crasemann Collins (2005). Eine Auseinandersetzung mit diesem Buch und dem von Flick (2005) wäre zur Analyse des ,Zeitgeistes“ von Raumordnung und Städtebau am Anfang des 20. Jahrhunderts sicher reizvoll, ist aber im Rahmen einer/dieser Rezension nicht möglich.
} 
Philipp Rapaport, seinem unterlegenen Konkurrenten um den Verbandsvorsitz des SVR, dem die Autorin einen kurzen Exkurs widmet (S. $131 \mathrm{f}$.), stammt, so wird damit doch eine kritische Sicht auf seine Persönlichkeit erkennbar, die immer mal wieder in verschiedenen Aussagen auftaucht. So äußerte der ehemalige Oberbürgermeister Essens und spätere Reichskanzler Hans Luther, der den SVR politisch durchsetzte und Robert Schmidt förderte: ,Er ist gegen seine Kollegen nicht nett gewesen. Wo er Gegnerschaft gegen seine Ideen witterte, war ihm die Bekämpfung mit allen Mitteln recht" (S. 17). Diese ambivalenten Einschätzungen Schmidts, „der vor Geist“ gesprüht, ,ein glänzendes Organisationstalent" besessen habe, aber von sich auch selbst sagte, man dürfe bei einem wichtigen Projekt ,auf eine Handvoll Privateigentümer und Hausbesitzer im Interesse des großen Ganzen keine Rücksicht nehmen“ (S. 16), lassen andeutungsweise erkennen, dass Robert Schmidt konzeptionell - nicht zuletzt als planerischer Visionär - und menschlich faszinierte, aber persönlich durchaus polarisieren konnte.

Robert Schmidt wurde 1869 in Offenbach geboren und ging in Frankfurt am Main zur Schule. Er war ein musisch veranlagter Mensch: „Die Geige im Gepäck war ihm wichtig, sie sollte ihn bis zu seinem Lebensende begleiten“ (S. 24). Er studierte ab 1889 in Hannover die Fächer Bauwesen und Wasserwirtschaft. Es folgte ,ein unkonventioneller Werdegang: vom Wasserbau-Ingenieur am Rhein zum Stadtbauinspektor in Essen“ (S. 25). Dort war ab 1902 die „Ausgestaltung der Stadt Essen zur Industrie- und Wohnstadt" seine Aufgabe, zuletzt - seit 1906 - in der Funktion eines Beigeordneten (S. 25 f.). Diese Funktion hatte er bis 1920, mit der Unterbrechung durch seinen Militärdienst von 1914 bis 1917, inne. Von 1920 bis 1932 war er der erste Direktor des SVR. 1913 wurde er mit seiner Denkschrift von 1912 an der Technischen Hochschule Aachen zum Dr.Ing. promoviert.

Weil ihm nicht „der Kohlenstaub des Reviers“ anhaftete, machte ihn dies ,,bei den Amtsleitern und Bürgermeistern der Revierstädte beinahe zu einer persona non grata“, zumal er Ideen propagierte, ,die aus lokaler Sicht nicht in die Reviermentalität passten“, die geprägt waren ,zunächst vom bäuerlichen Charakter und massiver Bodenständigkeit, dann aber fast ausschließlich vom Bergbau und von einer im neoliberalen [!] Wirtschaftsklima der Eisen- und Stahlproduktion mächtig gewordenen Lobby, die sich auf die lokalpatriotische Treue der Bergleute berufen zu können glaubte" (S. 29). So weit die Charakterisierung des Umfelds, in dem er wirkte. Wie gelang es ihm trotzdem, in diesem in jeder Hinsicht schwierigen, aber auch dynamischen und problemgeladenen räumlichen Umfeld Fuß zu fassen und sich zum anerkannten Experten zu entwickeln und dies in Zeiten, die gesellschaftlich und politisch durchaus turbulent zu nennen sind?
Ein großer Teil der vorliegenden Biographie ist die mit vielen Dokumenten gestützte Beschreibung „Im Dienst der Stadt Essen“ (S. 47 ff.), die zwei Jahrzehnte umfasst. Bemerkenswert ist in diesem Zusammenhang nicht nur, dass sich Schmidt von Anfang an für wohn- und lebenswerte Stadtteile mit einem hohen Anteil an ,Grün' und einer klaren Strukturierung der Verkehrswege einsetzte, wobei er sich im Einklang und in Kontakt mit internationalen Entwicklungen (Gartenstadtbewegung) befand, sondern er vertrat von Anbeginn an seine An- und Absichten auch ,geschickt gegenüber der Öffentlichkeit" (S. 48). So begleitete er als verantwortlicher Beigeordneter die Kruppsche Siedlung Margarethenhöhe. Es entstand eine auch international bemerkenswerte und beispielhafte „Garten(vor)stadt mit einem damals neuen Zusammenhang von Stadt und Landschaft“, die auch im Verbund mit einer „Wohnungsfürsorge als soziales Gebot" den Vorstellungen und Zielen Robert Schmidts entsprach (S. 58). Vorstellungen, die er nicht zuletzt im internationalen Austausch, wie bei den Städtebauausstellungen 1910 in Berlin-Düsseldorf-London bestätigt fand, wo auch die städtebaulichen Entwicklungen Essens internationale Beachtung fanden.

Für Ursula von Petz ist dies auch die Stunde der neuen Disziplin „Städtebau“, als einer, die nicht nur architektonisch geprägt ist, sondern als einer, die sich ,ihrer sozialen Verpflichtungen zunehmend bewusst“ wurde, bestimmt „,von den Gedanken an Natur und Grün [...] - nicht zuletzt unter dem Aspekt der Volksgesundheit" (S. 70). Dies war zu Beginn des 20. Jahrhunderts eine neue Perspektive, eine neue fachliche Synthese, deren Etablierung, gar Institutionalisierung eigentlich auch heute noch nicht abgeschlossen ist (jenseits aller integrierten Studiengänge beispielsweise), sondern sich immer wieder neu durchsetzen muss.

Aus dieser inhaltlich neuen Sicht auf Stadtentwicklung entstand die Denkschrift von 1912. Sie fußte auf den Unterlagen einer sogenannten Grünflächenkommission, einem Arbeitsausschuss, dessen Mitglied Schmidt war und deren Zusammenfassung oder Protokoll er ausarbeitete. Er war jedoch nicht Vorsitzender dieser vom Düsseldorfer Regierungspräsidenten eingesetzten Kommission. Er nutzte das Material der Kommission für seine Dissertation, ohne die Kommission und den Vorsitzenden zu fragen, worüber sich letzterer sehr erregte und die Arbeit als Plagiat einstampfen lassen wollte. Aber daraus entwickelte sich keine Affäre zum Nachteil Schmidts, denn der Regierungspräsident schrieb sogar ein Vorwort, zumal der Vorsitzende verstarb, „,infolge der Aufregung“, „wie seine Familie sagte“2. Es blieb dies letztlich eine ,Fußnote" in Schmidts Leben. Es zeigt aber auch, wie er in Verfolgung seiner Vorstellungen und Interessen - um der Sache willen? - durchaus bereit war, ungewöhnliche Wege zu gehen; ungewöhnlich wie die

\footnotetext{
2 So Josef Umlauf in einem Brief von 1959, dokumentiert auf S. 225.
} 
vorgelegte Dissertation selbst, denn sie ,kennt bedauerlicherweise keine Anmerkungen oder Literaturhinweise, aus denen sich die Diskussionen mit Kollegen als Inspirationsquellen für Schmidts Arbeit ermitteln ließen“ (S. 76). Sie war jedoch, wie Ursula von Petz mit Recht sagt, ,ein Meilenstein in der Planungsgeschichte des Reviers" - und mehr, merkt sie an -, denn er entwickelte ,,auf der Grundlage detaillierter statistischer Untersuchungen ein Siedlungskonzept, einen den Raum ordnenden regionalen Plan für eine von Ruß geschwärzte Industrieregion [...], für die unter Berücksichtigung der Standorte des Bergbaus sowie der Eisenund Stahlindustrie eine für die Zukunft geltende Ordnung [Hervorhebung im Original] angestrebt und propagiert wird, und zwar zum Wohle der dort lebenden und arbeitenden Einwohner“ (S. 79).

Ursula von Petz verweist darauf, dass Schmidts Denkschrift zwar auf der Arbeit der „Grünflächenkommission“ fußt, aber darüber hinausgehend gewissermaßen das Modell einer „Idealstadt“ vorlegt ,für eine Großstadt im Zeitalter der Industrialisierung, im Industrierevier des frühen 20. Jahrhunderts" (S. 93). Hier wird von ihr - vor dem Hintergrund der Denkschrift - in nuce eine Charakterisierung Schmidts vorgenommen, der sich in Verfolgung seiner Ideen und Vorstellungen die Quellen, die er aus der Wahrnehmung seines Amtes als vorhandene Informationen nutzte, zu eigen machte, wobei deren Herkunft und die Dokumentation ihrer Herkunft eher Petitessen waren, auf die Rücksicht zu nehmen er wohl nicht für nötig befand. Aber er kam damit als 43-jähriger etablierter Beigeordneter der nicht unbedeutenden Stadt Essen durch, obwohl die Inhalte der Denkschrift beim Regierungspräsidenten nicht unbedingt auf ungeteilte Zustimmung trafen. ${ }^{3}$ Hundert Jahre später ist angesichts der Bedeutung der Arbeit all dies wohl auch nur noch eine Petitesse, auch wenn es einen Blick auf die Person Schmidts wirft, die sich mit den zuvor genannten anderen Einschätzungen deckt. Insofern wird Ursula von Petz - zumal angesichts der Quellen - dem Anspruch einer Biographie gerecht. Sie hat keine Hagiographie geschrieben.

In einem weiteren Hauptteil ihres Buches geht es um Robert Schmidt ,[i]m Dienst des ,Siedlungsverbandes Ruhrkohlenbezirk ““ (S. 115 ff.). Im Dezember 1919 fand die Gründung des SVR statt und im Mai 1920 wurde Schmidt für eine Amtsperiode von 12 Jahren zum Verbandsdirektor gewählt (S. 119). Im Oktober 1920 legt er den Entwurf eines Arbeitsplanes vor. Auf der Agenda standen von Anfang an die Sicherung der regionalen Grünflächen im gesamten

\footnotetext{
3 Vgl. den im Buch dokumentierten „Abriss zu Leben und Werk Robert Schmidts" für eine geplante Denkschrift anlässlich seines 20. Todestages, die zwischen 1952 und 1955 von Prof. Josef W. Korte, RWTH Aachen, verfasst wurde und auf den S. 168-193 dokumentiert ist; hier S. 189.
}

Gebiet des SVR, aber auch die Schaffung von lebenswertem Wohnraum für die unter schwierigen Bedingungen Arbeitenden sowie der Ausbau der Verkehrsinfrastruktur auf Straße und Schiene. Schmidt dachte und handelte dabei „weitsichtig [...] in Jahrzehnten“ mit dem Ziel eines ,,räumlichen Ordnungskonzepts für das Industriegebiet zwischen Mörs [!], Duisburg und Dortmund“ (S. 139). Wobei zu berücksichtigen ist, dass es sich beim SVR damals nicht um eine Behörde, sondern um einen Verband handelte, der aufgrund seiner Struktur der Region ,institutionell eine demokratische Basis sichern" sollte (S. 146). Als Schmidt 1932 ausschied, wird andeutungsweise in einem Brief ablesbar, dass das schlechte Verhältnis Schmidts zu den Vertretern der Großstädte seiner Wiederwahl entgegenstand (S. 132). Nach seinem Ausscheiden kam der SVR in unsichere Fahrwasser und wurde in das NS-System eingepasst bzw. hatte während des Krieges andere Aufgaben als die der langfristigen Perspektive und Planung. Diese konnten erst wieder nach 1945 verfolgt werden - mit durchaus unterschiedlichen Organisationsstrukturen. „Zu diesem Zeitpunkt (nach 1945) waren jedoch die planerischen Grundlagen, insbesondere für die regionale Infrastruktur, durch die in den 1920er-Jahren geleistete Arbeit bereits festgelegt. Den Drachen ,Ruhrgebiet' hat Robert Schmidt nicht besiegen können. Doch als Geschlagener hat er den Kampfplatz nicht verlassen müssen“ (S. 147).

Abschließend darf aber nicht vergessen werden zu erwähnen, dass Robert Schmidt nicht nur lokal und regional tätig war - wenn auch nur beschränkt auf das Ruhrgebiet, denn ein mögliches Avancement nach Berlin verfolgte er nicht -, sondern er war auch national und international intensiv unterwegs. Er wurde 1922 zum ersten Präsidenten der neu gegründeten „Freien Deutschen Akademie des Städtebaus“ gewählt und im selben Jahr wurde ihm auch die Ehrenmitgliedschaft des „British Town Planning Institute" angetragen. Durch Teilnahme an internationalen Kongressen kam er mit bekannten Kollegen wie Ebenezer Howard, Lewis Mumford und Raymond Unwin in Berührung. Er beeindruckte eine Delegation der „Labour Party“, die 1927 das Ruhrgebiet besuchte. Stimmen aus dieser Delegation kamen zu dem kolportierten Schluss, dass Robert Schmidt „Lenin, Trotzky and Mussolini rolled into one“ ähnele - zur damaligen Zeit ein positiv treffendes Apercu. ,[A]nd he enjoyed the joke" wird als seine Reaktion überliefert (S. 151). Aber es wurde von englischer Seite auch angemerkt, dass er „,mit seiner deutschen Mentalität“, der Darstellung und Wahrnehmung seiner Rolle als Verbandsdirektor, sich keinen einzigen Monat in einer vergleichbaren britischen Funktion hätte halten können (S. 152).

Ursula von Petz beschließt ihre Biographie mit einem Verzeichnis der Publikationen Robert Schmidts (S. 157166) und einem umfangreichen Anhang (S. 167-226), in dem nicht nur die bereits erwähnte Skizze einer Biogra- 
phie von Josef W. Korte enthalten ist, sondern auch Briefe, Notizen und Interviews, in denen sich verschiedene Zeitgenossen zu Robert Schmidt äußerten, wie z. B. Hans Luther zur Nachfrage, welcher politischen Partei Robert Schmidt sich zugehörig gefühlt habe. Das Gerücht, er sei Kommunist gewesen; dies war ein 1933 von den Nazis kolportierter Verdacht. Es wurde aber auch behauptet, er habe ,das Mitgliedsbuch der SPD wie das des Zentrums“ (S. 197) gehabt. Luther meinte dazu: „Bob Schmidt hat die Politik als Sport betrieben“, er ,,behandelte sie alle ausgezeichnet: proletarisch und doch als grand seigneur" (S.197). Angemerkt sei, dass Hans Luther selbst parteilos war, aber im Umfeld der nationalliberalen Deutschen Volkspartei (DVP) politisch wirkte. Diese Form politischer Unabhängigkeit, aber auch bürgerlicher Verbindlichkeit verbunden mit einem großen Selbstbewusstsein scheint auch das nach außen prägende Bild Robert Schmidts gewesen zu sein, aber wohl auch sein Selbstbildnis. Dass er vor diesem Hintergrund nicht anfällig war für den aufkommenden Nationalismus und am Ende seines Lebens diesem durchaus skeptisch gegenüberstand, lässt dieser Auszug aus einem Brief vom 20. August 1933 an seine Schwester erkennen: „Es wird jetzt gar viel geschrien, aber das muß wohl sein, um das Volk anzuspannen. Mit klaren Gedanken wird arg Schindluder getrieben, nur um des Beifalls der Menge willen, wobei den langsam Denkern in fünf Minuten das Gegenteil serviert wird von dem was vorher gesagt war und zu beidem schreien sie bravo und heil! Das Alter macht offenbar skeptisch, weil man tiefer sieht" (S. 217). ${ }^{4}$ Am Ende seines Lebens hatte er in der Zeit des NS-Regimes ,nur mit Widerwärtigkeiten und Gehässigkeiten" umzugehen, wie er ebenfalls in einem Brief vom 4. November 1933 an seine Schwester schreibt (S. 217), aber er scheint im Kreise seiner Familie und im Praktizieren von Musik einen gewissen Frieden gefunden zu haben, bevor er am 19. Mai 1934 starb.

Jemand, der den Nationalsozialismus nicht als Erwachsener, sondern als heranwachsendes Kind, in der sogenannten Flakhelfer-Generation erlebt hat, hat für sich selbst von der „Gnade der späten Geburt“ gesprochen. Für Robert Schmidt ließe sich eher sagen, dass er die Gnade eines frühen Todes erfahren hat. Seine Haltung, seine Erfahrungen, seine internationale Reputation hätten nach 1945 eigentlich beste Anknüpfungspunkte für einen Neuanfang sein können. Dies hätte aber auch einen deutlichen Kontrast dargestellt

\footnotetext{
${ }^{4}$ Irgendwie scheint Robert Schmidt hier nicht nur eine genaue Charakterisierung damaliger populistischer Politik, sondern auch heutiger gelungen zu sein.
}

gegenüber den Belastungen oder Kontaminationen, denen sich die Überlebenden in aller Regel nicht stellten. Insofern wurde das Beispiel Robert Schmidts oder sein Erbe nicht aktiv wieder aufgenommen, wenn nicht gar verhindert (S. 21).

Eine Fülle von Dokumenten zu und unterschiedliche Einschätzungen über Robert Schmidt, aber wegen der Verluste im Krieg leider nur wenige, wenn auch sehr aussagekräftige persönliche Äußerungen, sind in dieser Biographie enthalten. Ursula von Petz hat versucht, aus allen Ecken Material zu Robert Schmidt zusammenzutragen und dies metikulös dokumentiert. Es entsteht das Bild eines Mannes, der sich vor dem Hintergrund seiner technischen Ausbildung und seiner musischen Ader sehr kundig und selbstbewusst in die Probleme einer Stadt wie Essen und dann des Ruhrgebiets insgesamt - in der Hochzeit der dort dominierenden Montanindustrie mit all den damit verbundenen Problemen und Herausforderungen der Siedlungsstruktur, die sich dem damals noch nicht bekannten Dreiklang der Nachhaltigkeit zu stellen hatten - eingearbeitet hat und sie selbstbewusst gestalten wollte. Es ist das Bild eines Mannes aus den Produkten seiner Arbeit und seinen Publikationen, verbunden mit einigen Erinnerungen und Einschätzungen, aber leider eben nur wenigen persönlichen Dokumenten. Leider wird auch der zeitgeschichtliche Hintergrund, den Robert Schmidt in seiner Lebens- und beruflichen Wirkenszeit durchlief, etwas zu wenig ausgeleuchtet. Insofern ist, was aber nicht das Versäumnis von Ursula von Petz ist, keine biographie intellectuelle Robert Schmidts - keine ,geistige Biographie ', um einen Begriff von Reinhard Bendix (1985) aufzunehmen entstanden, sondern eher eine biographie documentaire. Die Autorin versucht, bis in so vielen wie möglichen Einzelheiten wichtige Fakten und Einsichten über die Geschichte von Städtebau und Landesplanung in ihrer Entstehung in der Moderne im Spiegel von Robert Schmidts Lebenslaufs zu liefern. Sein Beitrag zu dieser positiven Entwicklung, die nach 1933 in Deutschland sehr schnell und auf sehr lange Zeit verspielt wurde, hätte nach 1945 am Anfang eines Neuanfangs stehen können, hätte dann aber wohl die Schattenseiten der Überlebenden grell ausgeleuchtet. Dieses unverbrauchte Erbe eines kreativen Visionärs und nicht angepassten Pragmatikers, auf dem wir fußen können, das nicht vergessen werden darf, von dem wir lernen können, in seiner Substanz und Fülle nachgezeichnet zu haben, dazu hat Ursula von Petz mit dieser Biographie dankenswerterweise beigetragen. 


\section{Literatur}

Bendix, R. (1985): Von Berlin nach Berkeley. Deutsch-jüdische Identitäten. Frankfurt am Main.

Crasemann Collins, C. (2005): Werner Hegemann and the Search for Universal Urbanism. New York/London.

Flick, C. (2005): Werner Hegemann (1881-1936). Stadtplanung, Architektur, Politik. Ein Arbeitsleben in Europa und den USA. Mün- chen. $=$ Einzelveröffentlichungen der Historischen Kommission zu Berlin 84.

Kastorff-Viehmann, R.; Utku, Y.; Regionalverband Ruhr (Hrsg.) (2014): Regionalplanung im Ruhrgebiet. Von Robert Schmidt lernen? Essen.

Schmidt, R. (1912): Denkschrift betreffend Grundsätze zur Aufstellung eines General-Siedelungsplanes für den Regierungsbezirk Düsseldorf rechtsrheinisch. Essen. 